\title{
Instrumentos para el análisis de la creación intelectual
}

\author{
María Margarita León Ortiz \\ Biblioteca Nacional José Martí, Cuba
}

\subsection{Resumen}

Se muestran los resultados obtenidos en la elaboración de procedimientos para el análisis de los textos de pensadores cubanos del siglo XIX, puestos en práctica en el análisis de las obras del presbítero Félix Varela y Morales. Mediante los instrumentos aplicados se logran identificar los núcleos temáticos abordados, las tendencias del pensamiento de los autores y se determinan las influencias presentes en sus obras. A partir de la indización profunda del contenido, la organización de la información en bases de datos y la utilización de las técnicas informétricas, entre otros, se obtienen resultados que se validan con los criterios de expertos y se posibilita la creación de un glosario, un diccionario de pensamientos del autor, un diccionario de personalidades citadas y el índice analítico de las obras estudiadas. (Autor)

Palabras clave: Análisis de información. Indización. Informetría. Metodologías. Creación intelectual.

\subsection{Abstract}

Results of an inquiry into the methodological procedures of the textual analysis of 19th century Cuban thinkers, particularly on the works of the priest Félix Varela y Morales. The methods used were in-depth content indexing, information structuring in a database, infometric analysis and external experts validation, among others. From their application, different products were obtained, mainly a glossary, a dictionary of the author's thoughts, a dictionary of cited personalities and the analytical index of the studied works. As a result, the author's intellectual themes, tendencies and influences are identified.

Keywords: Information analysis. Indexing. Informetrics. Methodology. Intellectual creation. 


\section{Introducción}

En el año 1998 se creó, en la Biblioteca Nacional José Martí, una línea de investigación orientada al análisis de la creación intelectual de los principales pensadores cubanos del siglo XIX, con el objetivo de establecer los núcleos temáticos de sus obras, las influencias recibidas y las tendencias de su pensamiento. A estos fines, fue elaborada una metodología para el análisis de la información en la creación intelectual, que preveía el logro de las vertientes antes apuntadas a través de la conformación de tres productos informativos: un índice analítico de las obras, un glosario que contuviera los principales conceptos definidos por el autor y un diccionario de citas. Una vez puestos en práctica los instrumentos diseñados en el análisis de las obras del Presbítero Félix Varela Morales y comprobada la efectividad de su aplicación, se generalizó su utilización en el conjunto de la obra de otros autores. En la actualidad estos procedimientos se están aplicando también en el análisis de la obra del filósofo y educador José de la Luz y Caballero.

Según las indagaciones efectuadas, que incluyó una intensa búsqueda bibliográfica y, muy especialmente, el intercambio con expertos en la obra vareliana e investigadores de la vida y obra de otras personalidades, no se encontraron indicios de que se haya estudiado la obra de intelectuales cubanos a partir de la utilización de técnicas propias de la actividad científico informativa, tenidas en cuenta en la elaboración de la metodología utilizada, ni a través de la indización profunda del contenido dirigida a la creación de los repertorios de referencia. Por estas razones puede considerarse la metodología que se presenta como un instrumento novedoso para abordar las obras de pensamiento.

\section{Desarrollo}

La elaboración de la metodología tuvo en cuenta los siguientes principios teóricos:

- Uno de los objetos de estudio de las Ciencias de la Información es el llamado procesamiento de la propia información y ...

- esta acción se lleva a efecto a través de los procesos de análisis y de síntesis, que no son más que "procesos del pensar o la descomposición real de un todo en sus partes y de la reunificación de un todo a base de sus partes" (Rosenthal, 1973).

- La realización del análisis lleva implícito el estudio de determinados componentes del objeto y el estudio de su contenido semántico, que permitan describirlo de forma exhaustiva y profunda a través de la indización. 
- La indización “... implica la necesidad de prestar atención a todos los elementos presentes en el contenido semántico del documento, que puedan ser de interés a los destinatarios finales ..." (Vizcaya, 1997a), y, por tanto, ...

- la asignación de un conjunto de varios términos a la fuente procesada, con la suficiente profundidad en la indización, brinda posteriormente la posibilidad de combinar los términos de interés, a través de la poscoordinación de elementos.

- A medida que la descripción de los temas tratados en un documento es más profunda y exhaustiva, puede llevarnos a una reformulación normalizada de todo el documento.

A partir de estos elementos de razonamiento, se efectuó el análisis de contenido de las 744 páginas de las obras de Félix Varela que incluyeron:

- Varias proposiciones para el ejercicio de los bisoños.

- Instituciones de filosofía ecléctica para uso de la juventud.

- Elenco de 1816.

- Instrucciones morales y sociales.

- Lecciones de filosofía.

- Miscelánea filosófica.

- Observaciones sobre la Constitución política de la Monarquía española.

- El Habanero, papel político, científico y literario.

- Cartas a Elpidio sobre la impiedad, la superstición y el fanatismo, en sus relaciones con la sociedad.

- Discursos.

- Epistolario.

\subsection{Etapas previstas en la metodología}

\subsubsection{Primera etapa}

- Consulta de la bibliografía relacionada con la confección de índices analíticos, la elaboración de diccionarios de citas y de glosarios.

- Estudio de la bibliografía relacionada con las técnicas de investigación, indización y sobre lenguajes documentales.

- Revisión de numerosos repertorios publicados.

- Lectura de biografías y otros documentos históricos.

- Estudio de los textos del autor objeto de investigación.

- Consulta de la bibliografía pasiva relacionada con cada obra que se analiza. 


\subsubsection{Segunda etapa}

1. Indización del contenido de los textos, a partir de la extracción de :

- Palabras clave: Aquellas palabras que posean un significado relevante dentro del texto.

- Nombres personales: Las personas mencionadas, a saber, autores de las citas, científicos, gobernantes, hombres de letras, personajes de ficción, etc.

- Entidades: Incluyen instituciones, órganos de gobierno, expediciones, etc.

- Acontecimientos históricos: Todas aquellas menciones referidas a sucesos históricos de diferente índole.

- Títulos: Los correspondientes a libros, así como los que identifican cada artículo o división interna de las obras.

- Lugares: Referencias a ciudades, países, continentes, sitios, etc., así como todas las regiones geográficas.

- Conceptos: Aquellas definiciones dadas sobre diferentes tópicos.

- Pensamientos: Fragmentos de textos que representan ideas expresadas por el autor sobre distintos temas.

- Lecturas: Todas las citas bibliográficas y lecturas presentes tanto implícita (que se infieren del contexto) como explícitamente, que sirven como apoyatura al desarrollo del pensamiento del autor.

- Publicaciones: Las revistas y periódicos mencionados.

2. Eliminación de la sinonimia y polisemia.

3. Indicación de referencias a los términos seleccionados.

4. Asignación a cada palabra clave, entidad, acontecimiento histórico, concepto y pensamiento de una materia general de acuerdo con el contexto, el ámbito de desarrollo o el terreno en que éste tuvo lugar, respectivamente. En cuanto a los conceptos y pensamientos, se les indiza con materias generales según el contenido expresado. En nuestro caso, se seleccionan a partir de las diferentes corrientes identificadas en el pensamiento del autor, tomando como base el Epigrafiario de la Biblioteca Nacional.

5. A los pensamientos se les indican, además, puntos de acceso alternativos mediante el uso de palabras o expresiones propias del autor, e indicativas de las características de su discurso y de la expresión de sus ideas. A 


\begin{tabular}{|l|c|c|}
\hline Elementos de indización & 994 & 2134 \\
\hline Palabras clave & 371 & 588 \\
\hline Nombres personales & 315 & 464 \\
\hline Pensamientos & 167 & 531 \\
\hline Lugares & 146 & 154 \\
\hline Conceptos & 133 & 133 \\
\hline Títulos & 115 & 169 \\
\hline Lecturas & 70 & 122 \\
\hline Entidades & 66 & 66 \\
\hline Referencias & 15 & 18 \\
\hline Acontecimientos históricos & 7 & 7 \\
\hline Personajes de ficción & 6 & 6 \\
\hline Personajes mitológicos & 5 & 5 \\
\hline Personajes bíblicos & 5 & 17 \\
\hline Publicaciones seriadas & $\mathbf{2 4 1 5}$ & $\mathbf{4 4 1 4}$ \\
\hline TOTAL & & \\
\hline
\end{tabular}

Tabla 1. Resultados obtenidos en la primera etapa

estos términos se les denomina "temas" para diferenciarlos de las palabras clave y las materias utilizadas.

6. Búsqueda bibliográfica para la recolección de datos que permitan conocer y caracterizar a las personas reales, los personajes de ficción, las entidades, los acontecimientos históricos y las publicaciones seriadas mencionadas en las obras, y en especial, tratar de establecer el motivo de su mención. Con los datos obtenidos se elaboran fichas biográficas o de contenido, según el caso.

7. Se aplica el mismo procedimiento en la investigación de los elementos necesarios para completar las citas bibliográficas del autor y de las lecturas identificadas en las obras (Tabla1).

\subsubsection{Tercera etapa}

1. Diseño de una base de datos en CDS/ISIS, con formato propio, para la carga de los datos seleccionados. (Ver Anexo 1) 
2. Introducción de los datos obtenidos, utilizando el WINISIS, debido a las facilidades que brinda el ambiente Windows para la realización de diferentes tareas simultáneamente y teniendo en cuenta que se pudiera contar con versiones electrónicas del texto de las obras.

\subsubsection{Cuarta etapa}

(Aplicación de los estudios métricos a cada una de las obras por separado para arribar a conclusiones)

1. Selección de las variables a utilizar para los análisis métricos. Estas variables se ajustarán a las características específicas del autor y de los textos objeto de análisis.

2. Realización de búsquedas por diccionario o booleanas en la base de datos, utilizando las variables previstas.

3. Confección de índices en ISIS con el resultado de las búsquedas efectuadas.

4. Creación de hojas de trabajo en Microsoft Excel con los ficheros texto de los índices.

5. Confección de tablas de frecuencia en Excel.

6. Análisis e interpretación de los resultados de las mediciones.

7. Confrontación de los resultados obtenidos con los puntos de vista de expertos en la literatura especializada.

\subsection{Variables utilizadas}

Para identificar las tendencias temáticas existentes en las obras, se efectúa un análisis de los resultados de las mediciones aplicadas a las materias aplicadas a las palabras clave, entidades y acontecimientos históricos, así como la clasificación por materias de los pensamientos y conceptos seleccionados y los temas indicados como puntos de acceso alternativos (Tabla 2).

Otros elementos a tenidos en cuenta como variables para el análisis temático son:

\begin{tabular}{|l|c|c|}
\hline Otros elementos de indización & Cantidad & Ocurrencias \\
\hline Materias & 20 & 3156 \\
\hline Temas & 228 & 630 \\
\hline TOTAL & $\mathbf{2 4 8}$ & $\mathbf{3 7 8 6}$ \\
\hline
\end{tabular}

Tabla 2

Scire. $6: 2$ (jul.-dic. 2000) 31-47. 
- Ámbito de desarrollo o tipo de actividad realizada por las personas mencionadas en las obras,

- nacionalidad de dichas personas y ...

- lugares mencionados.

Si se toma como universo la suma de ocurrencias de los elementos de indización de las Tablas 1 y 2, se obtiene como resultado que se tomaron en consideración 8.200 términos para el análisis de contenido de las 744 páginas de las obras, lo que proporciona una densidad de indización de 11 términos promedio por página. Esto evidencia la profundidad lograda en el análisis de los textos.

Las influencias presentes en cada obra se identifican a través del análisis de las personas mencionadas, los autores citados, la época en que realizaron sus actividades, su nacionalidad, las lecturas evidenciadas en los textos, los títulos de las obras mencionadas, las entidades, los lugares y los acontecimientos históricos.

Para el establecimiento de los núcleos temáticos fundamentales, se utilizan las dos variables previstas que reflejan las categorías de contenido: materias y temas. Aquéllas que presenten mayor frecuencia en sus respectivas tablas, representan la mayor concentración de contenido.

\subsection{Acerca de los productos informativos elaborados}

Para facilitar la organización de la información requerida para la elaboración de repertorios, estos datos se estructuraron en cuatro grupos según su destino: índice analítico, diccionario de citas, glosario y lecturas (Tabla 3).

\subsubsection{Indice analítico}

Con el objetivo de facilitar la consulta de las obras el índice analítico previsto (Véase el anexo 2), se incluyeron los siguientes puntos de acceso: materias generales, palabras clave, nombres personales, acontecimientos históricos, títu-

\begin{tabular}{|l|c|}
\hline $\begin{array}{l}\text { Componentes de la } \\
\text { base de datos }\end{array}$ \\
\hline Índice analítico & 1840 \\
\hline Diccionario de citas & 315 \\
\hline Glosario & 146 \\
\hline Lecturas & 115 \\
\hline Total & $\mathbf{2 4 1 6}$ \\
\hline
\end{tabular}

Tabla 3

Scire. $6: 2$ (jul.-dic. 2000) 31-47. 
los, lugares, personajes, términos que responden a pensamientos o conceptos y referencias de véase (para hacer envíos de los términos no usados a los utilizados) y de véase también (para indicar la relación entre diferentes términos).

\subsubsection{El glosario y el diccionario de citas}

El glosario resultante de la investigación, donde se encuentran representados los conceptos o definiciones dados por el autor a diferentes tópicos, se ajusta a la segunda definición de la Real Academia de la Lengua Española sobre este término, es decir: "Catálogo de palabras de una misma disciplina, de un mismo campo de estudio, etc., definidas o comentadas" (1994, t. 1, p. 10).

En el proceso de concepción, tanto del glosario como del diccionario de citas (Véase Anexo 2), se utilizó la definición dada por la Dra. Dolores Vizcaya (1997a, p. 58-60) de artículo léxico del descriptor: "unidad del diccionario semántico que consta de un descriptor principal y de las unidades léxicas relacionadas a él por determinadas relaciones paradigmáticas", pero con una nueva connotación, por cuanto los artículos léxicos están estructurados en registros de una base de datos.

A cada artículo léxico del diccionario de citas o glosario le corresponde un término principal que responde a la definición dada por la autora mencionada (1997b, p. 156) sobre descriptor: "Término (palabra o expresión) que se ha escogido, a partir de un conjunto de sinónimos, de cuasisinónimos y de términos emparentados, para representar, de manera unívoca, un concepto susceptible de intervenir en los documentos y en las consultas que se examinan dentro de un sistema documental dado, e incluido por tanto dentro del tesauro de descriptores de ese sistema". Los términos principales seleccionados en un principio como palabras clave, pasaron por un proceso de depuración, para convertirlos en descriptores, y así representar de manera unívoca los conceptos o citas asociados a ellos, tomados de los textos de las obras.

Las unidades léxicas que se relacionan a estos descriptores principales (términos) son:

- El concepto o los pensamientos,

- Los términos genéricos: las materias generales asignadas a cada pensamiento o concepto,

- Los términos relacionados: están constituidos por los denominados temas, que son palabras extraídas de los pensamientos y, como se explicó con anterioridad, constituyen puntos de acceso alternativos para recuperarlos. Estos términos están relacionados con el descriptor principal por el propio contenido de dichos pensamientos y dicha relación se expresa a través de referencias de véase también. En el caso del glosario no aparecen términos 
relacionados porque en él existe un sólo término al que corresponden una o varias definiciones que fueron clasificadas por la materia general que le corresponde,

- Términos no usados: referencias de véase utilizadas, entre otros fines, para controlar la sinonimia,

- La remisión a la ubicación del concepto o los pensamientos dentro de la totalidad de la obra de Varela.

En este caso no se establecen las relaciones paradigmáticas entre el descriptor principal y las unidades relacionadas a él, ya que según el concepto manejado, éstas "son las relaciones lógicas (de asociación) que se establecen entre las palabras antes del momento de su utilización y que no dependen del contexto en el cual se empleen" (ibidem, p. 159). De acuerdo con lo expresado con anterioridad, las relaciones de asociación que se establecen en este caso, están dadas por el contenido de los pensamientos o el concepto en el momento de su utilización.

\subsubsection{Diccionario de personalidades}

Por último, con los elementos obtenidos como resultado de la búsqueda de los datos biográficos para la identificación de las personas (y para descifrar el motivo de su mención en los textos) se obtiene, como un producto adicional de la investigación, un diccionario de personalidades.

\section{Conclusiones}

Las principales conclusiones obtenidas de la investigación son las siguientes:

- La metodología confeccionada permitió arribar a conclusiones relativas a las tendencias temáticas y las influencias presentes en las obras analizadas.

- Se comprobó su factibilidad en la elaboración de repertorios de referencia, con auxilio de las técnicas de cómputo y de las propias de los estudios métricos.

- Se comprobó que los procedimientos y los instrumentos utilizados como complemento de dicha metodología son recomendables en investigaciones similares relativas a otras personalidades de la cultura cubana y universal.

- El análisis y la síntesis aplicados a obras de pensamiento, conjugados con la aplicación de técnicas informétricas y bibliométricas, permitieron llegar a conclusiones y validar, desde un ángulo diferente, aquéllas obtenidas por otros medios.

- Se demostró que con el auxilio de las nuevas tecnologías se pueden elaborar repertorios de referencia, con mayor rapidez y cualitativamente superiores a los realizados por métodos tradicionales. 


\section{Anexo 1. Base de datos de obras de Varela}

\begin{tabular}{|c|c|}
\hline $\begin{array}{l}\text { Identif. de } \\
\text { campo }\end{array}$ & Contenido \\
\hline 1 & $\begin{array}{l}\text { Indica el destino de los datos y su formato de salida para índice analítico, glosario y } \\
\text { diccionario de citas. }\end{array}$ \\
\hline 2 & $\begin{array}{l}\text { Palabras o frases significativas: Nombres personales, palabras claves, lugares, } \\
\text { acontecimientos históricos, términos que distinguen pensamientos o conceptos, etc. }\end{array}$ \\
\hline 3 & Abreviatura indicativa del tipo de término: Ej. NP (Nombre personal) \\
\hline 4 & $\begin{array}{l}\text { Datos sobre personas, acontecimientos históricos, etc. de interés para el diccionario } \\
\text { de personalidades o para la investigación en general. }\end{array}$ \\
\hline 5 & Actividad principal en que se desarrolla una personalidad. Ej. Filósofo \\
\hline 6 & Siglo de florecimiento. \\
\hline 7 & Lugar de origen o nacionalidad \\
\hline 8 & $\begin{array}{l}\text { Texto de los conceptos o pensamientos seleccionados, asociados al término del } \\
\text { campo } 2 \text {. }\end{array}$ \\
\hline 9 & $\begin{array}{l}\text { Palabras significativas del texto de los pensamientos que actúan como puntos de } \\
\text { acceso alternativos a dichos textos. }\end{array}$ \\
\hline 10 & $\begin{array}{l}\text { Materias generales que se utilizan para indizar los textos de los pensamientos, } \\
\text { definiciones, palabras claves y acontecimientos históricos. }\end{array}$ \\
\hline 11 & Término no autorizado por ser de difícil comprensión o sinónimo. \\
\hline 12 & Término al que se remite o autorizado \\
\hline 13 & $\begin{array}{l}\text { Ubicación dentro de las obras: ^aAbreviatura de la obra en particular. ^bTomo de la } \\
\text { edición de las Obras completas del autor. ^cPágina o páginas donde se localiza el } \\
\text { término. }\end{array}$ \\
\hline 14 & $\begin{array}{l}\text { Término relacionado que se desea enlazar en el índice con el término que aparece } \\
\text { en el campo } 2 \text {. }\end{array}$ \\
\hline 15 & $\begin{array}{l}\text { Relación de términos relacionados con el término principal a través de véase o véase } \\
\text { también. }\end{array}$ \\
\hline 16 & Fechas de cartas, discursos, etc. \\
\hline 17 & Persona o personas a quienes se dirije una carta. \\
\hline 18 & Datos descriptivos de las obras, según aparecen citadas por el autor. \\
\hline 19 & Datos descriptivos de las obras una vez realizada la investigación correspondiente. \\
\hline 20 & Texto de la cita de la obra (opcional) \\
\hline 21 & $\begin{array}{l}\text { Lectura realizada por el autor y que se infiere por el contexto. Ej. Mención de un } \\
\text { personaje de ficción, una frase, una reflexión, etc. }\end{array}$ \\
\hline
\end{tabular}

Tabla 4. Base de datos: Identificación y contenido de los campos

Scire. 6 : 2 (jul.-dic. 2000) 31-47. 
01 SALIDA:

02 TERMINO:

03 IDENT. TERMINO:

04 DATOS:

05 ACTIV.:

06 SIGLO:

07 NACIONALIDAD:

08 CONCEPTO O PENSAMIENTO:

09 TEMAS:

10 MATERIAS:

11 TERMINO NO USADO:

12 VEASE:

13 FUENTE:^^a $\mathbf{a}^{\wedge} \mathbf{b}^{\wedge} \mathbf{c}$

14 VEASE TAMBIEN:

15 REFERENCIAS EN:

16 FECHA:

17 DESTINATARIO:

18 CITA BIBLIOGRAFICA ABREVIADA:^ $\mathbf{a}^{\wedge} \mathbf{b}^{\wedge} \mathbf{c}$

19 CITA BIBLIOGRAFICA COMPLETA: $\mathbf{a}^{\wedge} \mathbf{b}^{\wedge} \mathbf{c}$

20 TEXTO DE LA CITA:

21 MENCION DE LECTURA: ${ }^{\wedge} \mathbf{a}^{\wedge} \mathbf{b}^{\wedge} \mathbf{c}$

Fig. 1. Base de datos: formato de entrada

Scire. $6: 2$ (jul.-dic. 2000) 31-47. 


\section{Anexo 2}

\section{1. Índice analítico (Muestra)}

AUXILIOS EXTRANJEROS; Hab., II, 200, 245, 248, 249

Ayacucho (Fragata); Hab., II, 286

AYUNTAMIENTOS; Obs., II, 36, 37, 60, 61, 62, 64, 69

AZUCAR; Hab., II, 279, 286

BENEFICENCIA; Obs., II, 65

Bertrando, Conde; Obs., II, 36

Bielefeld, Jacobo Federico, Barón de; Obs., II, 19

BIEN; Obs., II, 13

Bolívar, Simón; Hab., II, 184, 231, 266, 278, 284

Bombas habaneras; Hab., II, 183

Borbón, Luis Antonio de; Hab., II, 212

Borbones; Obs., II, 15

Boston; Hab., II, 241

Bourmont, General v. Ghaisnes, Luis Augusto Víctor de

Brasil; Hab., II, 224, 225, 284

Bravo, Nicolás; Hab., II, 266

Buenos Aires; Hab., II, 190, 224

BULAS PONTIFICIAS; Obs., II, 40, 55

Burdeos; Hab., II, 231

CABILDOS; Obs., II, 37

Cádiz; Hab., II, 205, 211, 212, 281

CAFE; Hab., II, 279, 286

El Callao, Rendición de; Hab., II, 271, 278

CALUMNIA; Hab., II, 175, 177, 185

CAMARAS; Obs., II, 30, 31, 33, 38

Cambia-colores; Hab., II, 149

CAMBIO DE OPINION; Hab., II, 151

CAMPAÑAS; Hab., II, 160

Campeche, Puerto de; Hab., II, 171

Canning, George; Hab., II, 215, 281

Canterac, José; Hab., II, 184, 271

CAPITALES EXTRANGEROS [sic]; Hab., II, 164

CAPITALISTAS; Hab., II, 198, 200

Capmany Surís y de Montpalau, Antonio; Obs., II, 45

Carlos V; Hab., II, 247

Carta a un amigo respondiendo a algunas dudas

ideológicas; Hab., II, 192 


\subsection{Glosario (muestra)}

\begin{abstract}
ATEISMO
El ateísmo no puede pasar de una duda, y que darle el carácter de una doctrina fundamental y norma de operaciones en el más importante de todos los negocios, no puede ser sino efecto de pasiones desarregladas.
\end{abstract}

Car.Elp.1, III , 6

\section{CIENCIA DE LA POLITICA}

... es la ciencia que comprende los derechos recíprocos de los que gobiernan y de los que son gobernados.

Obs., II, 19

\section{DIPUTADOS}

Diputado quiere decir lo mismo que enviado por una provincia, con facultades para representar derechos y proponer mejoras, de manera que es un verdadero apoderado de la provincia, pero que al mismo tiempo lo es de toda la nación en virtud de sus leyes fundamentales...

Obs., II, 41

\section{ENEMIGOS DE LA LIBERTAD}

... esos mismos hombres que, validos de su influjo, procuran por todos medios separar de la opinión común el círculo más o menos extenso que manejan; esos mismos hombres para quienes la unión, como no fuese en sufrir las cadenas de un gobierno despótico, sería el mayor de los males, que tratan de evitar por todos los medios; esos mismos están continuamente deplorando la desgraciada desunión de los partidos.

Hab., II, 253

\section{ESCLAVO}

Cuando el hombre no depende de la ley, sino de la libre voluntad o del capricho del que le gobierna, es esclavo por más dulce que se finja su esclavitud.

Hab., II, 280

\section{IDEOLOGIA}

El objeto de esta ciencia es reducir las ideas del hombre a su verdadero origen, indicando los pasos con que se fueron desenvolvimiento las facultades intelectuales y morales, y la relación de los conocimientos adquiridos.

Ora., I, 87

\section{IRA SANTA}

... es la que excita al espíritu arreglado la vista del crimen y la obstinación del criminal.

Sent., I, 96

\section{LOGICA}

... la ciencia de dirigir el entendimiento.

Lec., I, 141

\section{LOGICA NATURAL}

... consiste en la facilidad de percibir los errores por la luz de la razón ...

Epi., III, 235

\section{MASCARAS POLITICAS}

... encubren al hombre en la sociedad, y le presentan con un semblante político muy distinto del que realmente tendría si se manifestase abiertamente.

Hab., II, 145

\section{OFICIOS PERFECTOS}

Los oficios perfectos, que son los que propiamente pertenecen a la justicia, se fundan en estas dos máximas: $1^{\circ}$ dar a cada cosa lo que tiene derecho de exigir de nosotros; $2^{\circ}$ no hacer a otro lo que no quisiéramos se nos hiciese.

Elenco 1816, I, 81

\section{SENTIR}

... un acto de la sensibilidad que consiste en percibir simplemente una idea, y éste no es el de juzgar, ni el de desear, pues precede a ambos y podemos llamarse simplemente sentir.

Misc., I, 323 


\subsection{Diccionario de citas (Muestra)}

\section{ACALORAMIENTO}

El acaloramiento es un signo muy equívoco, pues acompaña al justo que defiende lo recto, $\mathrm{y}$ al perverso que quiere cohonestar su perversidad, al filósofo que sostiene los derechos de razón, y al preocupado que se empeña en sostener quimeras.

Lec., I, 170.

Misc., I, 353

\section{ACCIONES}

... las acciones como las palabras, deben disponerse en el orden analítico en que se adquirieron las ideas. Cuando uno sabe accionar en dicho orden, es muy fácil entenderle; así como el que habla disponiendo en el mismo orden sus palabras, tiene un lenguaje claro. Lec., I, 164

\section{ACTOS DE NUESTRA SENSIBILIDAD}

Tenemos, pues, cuatro actos de nuestra sensibilidad bien distinguidos: sentir simplemente, acordarse, juzgar y querer...

Misc., I, 323

\section{Adulación véase FRANQUEZA}

\section{ALEGRIA}

La alegría excita nuestro ánimo por la contemplación de un bien, y produce gratas emociones, teniendo diversos grados según la naturaleza de dicho bien, y el conocimiento que adquirimos de su utilidad, esto es, de sus relaciones con nosotros.

Lec., I, 244

La alegría exalta el alma, y es como el gran resorte de sus operaciones; mas cuando es excesiva llega a trastornar el espíritu, y da cierta ligereza opuesta a la madurez y buen juicio.

Sent., I, 97

\section{AMERICANO}

$\mathrm{El}$ americano oye constantemente la imperiosa voz de la naturaleza que le dice: yo te he puesto en un suelo que te hostiga con sus riquezas y te asalta con sus frutos; un inmenso océano te separa de esa Europa, donde la tiranía ultrajándome, holla mis dones y aflige a los pueblos; no la temas: sus esfuerzos son impotentes; recupera la libertad de que tú mismo te has despojado por una sumisión hija más de la timidez que de la necesidad; vive libre e independiente; y prepara un asilo a los libres de todos los países; ellos son tus hermanos. Hab., II, 189

\section{AMISTAD}

La amistad es el bálsamo del desconsuelo, y la comunicación de ideas el alivio de las almas sensibles.

Car.Elp.1, III, 5

Si la casa de un amigo empezase a arder, cuando él reposa tranquilo, ¿sería prudencia y amistad, no exci- tarle del sueño, no advertirle el peligro, bajo pretexto de no asustarle, de no causar un trastorno en su familia, de no exponerle a las pérdidas inevitables que ocasiona una pronta salida?

Hab., II, 239

\section{Amor véase IMPIEDAD; JUVENTUD}

\section{COLONIAS}

Los pueblos que por su debilidad se hallan en el triste estado de colonias, esto es, en el de producir para los goces de otro más fuerte, sólo pueden soportar esta desigualdad social, en virtud de una recompensa que encuentran en la protección y garantía que se les presta ...

Hab., II, 173

\section{DINERO}

Bien sé que el dinero es el señor del mundo y que los que sólo tienen ideas mundanas siempre piensan en dinero.

Car.Elp.2, III, 120

\section{ESTADOS UNIDOS}

Siendo considerado este pueblo como norma de la tolerancia religiosa, es preciso no formarnos ideas equivocadas acerca de él, porque al fin desaniman a sus imitadores, cuando la experiencia les demuestra que no han llegado y que acaso es imposible llegar a una perfección imaginaria, que toman por existente. Car.Elp.1, III, 57

Siempre se presenta a este pueblo como un modelo de perfección, y aunque yo soy uno de sus admiradores, quisiera igualmente que no se alucinasen muchos y perdiesen la importante lección que la experiencia puede darles en este mismo país que tanto elogian. Car.Elp.1, III, 84

\section{INDEPENDENCIA}

... la independencia de la isla de Cuba no es un objeto de elección sino de necesidad, pues un hado político la decreta, y que los que la presentan obstáculos no hacen más que privarla de los bienes de que podría estar acompañada ...

Hab., II, 225

La independencia y libertad nacional son hijas de la libertad individual, y consisten en que una nación no se reconozca súbdita de otra alguna, que pueda darse a sí misma sus leyes, sin dar influencia a un poder extranjero, y que en todos sus actos sólo consulte a su voluntad, arreglándola únicamente a los principios de justicia, para no infringir derechos ajenos. Obs., II, 18

Véase además AMERICANO; ISLA DE CUBA INGENUIDAD 


\subsection{Diccionario de personalidade en la obra vareliana (muestra)}

Aner de Esteve, Felipe: Diputado a las Cortes de Cádiz en 1812, representando a Cataluña.

Obs., II, 43, 63

Arango, Rafael: Nace en La Habana en 1788 y muere el 6 de noviembre de 1850. Coronel efectivo de los Reales Ejércitos, Caballero de la Orden de San Fernando, y uno de los héroes del 2 de mayo. Con experiencia militar se retira a Cuba en 1821 y forma parte de la Junta Militar.

Hab., II, 234

Argüelles, Agustín de: Hombre de estado español y distinguido orador asturiano. Nace en Oviedo el 18 de agosto de 1776 y muere en Madrid el 26 de marzo de 1844. Representó a la provincia de Oviedo en las Cortes de Cádiz, donde fue el campeón de las ideas liberales. Se encargó de la redacción del preámbulo y articulado de la Constitución de 1812, en defensa de la cual pronunció sentidos discursos que le valieron el dictado hiperbólico de Divino Argüelles.

Obs., II, 40, 61, 63

Averroes: Médico, jurista y filósofo árabe. Nació en Córdoba (1126-1198). Comentarista de Aristóteles, inclinábanse sus doctrinas filosóficas hacia el materialismo y el panteísmo, por lo que fueron condenadas por la Universidad de París y por la Santa Sede. Misc., I, 418, 420

Bielefeld, Jacobo Federico, Barón de: Publicista alemán. Nace en Hamburgo en 1716 y muere en 1770. Estuvo al servicio de Federico El Grande, cuando este era sólo Príncipe Real de Prusia. Después desempeñó algunos cargos diplomáticos, y en 1747 fue nombrado inspector general de las universidades prusianas.

Obs., II, 19

Borbón, Luis Antonio de: Nace en Versalles el 8 de agosto de 1775 y muere el 3 de junio de 1844 en Gorz. En 1823 se le confiere el mando superior del ejército que fue a España a restablecer el gobierno absoluto. Mandaba al ejército de los Cien Mil Hijos de San Luis.

Hab., II, 212

Bravo, Nicolás: General mexicano. Nace en Chilpancingo entre 1784 y 1790 y muere en 1854 .

En 1811 se unió al movimiento revolucionario del que no tardó en ser uno de los más distinguidos.

Obs., II, 266

Canning, George: Célebre estadista y orador inglés. Nace en Canning el 11 de abril de 1770 y muere en Chiswick, Londres, el 8 de agosto de 1827.
Favoreció la insurrección de las colonias españolas en América.

Hab., II, 215, 281

Cicerón, Marco Tulio: Político, orador, filósofo y literario de la antigua Roma. Nace el 3 de enero de 647.

Lec., I, 142, 191, 196, 213, 249, 260, 270

Clemente V: Papa de 1305 a 1314. Trasladó la Santa Sede a Aviñón y disolvió la Orden de los Templarios para dar satisfacción a su protector Felipe El Hermoso de Francia.

Elenco 1816, I, 75

Descartes, René: Filósofo y científico (1596-1650). Sus principales obras fueron Ensayos filosóficos (1637), Meditaciones metafísicas (1641), Los principios de la Filosofía (1644).

Misc., I, 321, 322, 340, 406, 407, 418

Estanislao III: Rey de Polonia. Nace en Lituania el 17 de enero de 1732 y muere en San Petersburgo el 12 de febrero de 1798. En 1764, Catalina de Rusia hace elegir por la fuerza de las armas a Estanislao III como sucesor de Augusto III.

Hab., II, 218

Foción: Nació hacia el año 400 a.J.C. y muere en 318. Tomó parte de las campañas de Cabria, mandaba en la batalla de Nasos y se ganó en Atenas, y fuera de ella, tal confianza que fue elegido estratega 45 veces. Al propio tiempo no era amigo de la democracia reinante y permaneció fiel a esta política hasta después de la muerte de Alejandro. Al disolverse la constitución democrática tomó la dirección del Estado, hasta que fue derribado. En el 318 fue acusado de traición y condenado a muerte, obligándole a tomar veneno.

Ora., I, 10

Grocio, Hugo: Teólogo y jurisconsulto holandés (1583-1645). Fue con el español Vitoria uno de los precursores del derecho internacional.

Car.Elp.1, III, 11

Guadalupe Victoria, Manuel Félix Fernández: Primer presidente de la República constitucional de México (1780-1843). En 1824 es elegido para la presidencia de la República, cargo que desempeñó hasta 1829. Durante este tiempo llevó a cabo un tratado con Inglaterra, redujo las fiestas nacionales y religiosas, se rindió el Castillo de Ulúa (último refugio de los españoles), se hizo efectiva la abolición de la esclavitud, declaró fiesta nacional el 5 de febrero en memoria de San Felipe de Jesús y dio la ley sobre la expulsión de los españoles.

Hab., II, 231, 266

Scire. $6: 2$ (jul.-dic. 2000) 31-47. 


\subsection{Abreviaturas utilizadas}

\begin{tabular}{|c|c|}
\hline Abreviatura & Contenido \\
\hline Car.Elp.1 & Cartas a Elpidio. Impiedad \\
\hline Car.Elp.2 & Cartas a Elpidio. Superstición \\
\hline Elenco 1816 & Elenco de 1816 \\
\hline Epi. & Epistolario de Félix Varela \\
\hline Hab. & El Habanero, papel pol ítico, científico y literario \\
\hline $\begin{array}{l}\text { Instrucción } \\
\text { pública }\end{array}$ & Escritos varios (1826-1830): Instrucción Pública \\
\hline Lec. & Lecciones de filosofía y otros escritos filosóficos \\
\hline Misc. & Miscelánea filosófica \\
\hline Obs. & $\begin{array}{l}\text { Observaciones sobre la Constitución política de } \\
\text { la Monarquía Española }\end{array}$ \\
\hline Ora. & Oratoria de Félix Varela \\
\hline Sent. & $\begin{array}{l}\text { Fragmentos de las sentencias o instrucciones } \\
\text { morales y sociales para la juventud escritas por } \\
\text { Don Félix Varela y Don Justo Vélez a instancias } \\
\text { de la Real Sociedad Patriótica de La Habana } \\
\text { (1818) }\end{array}$ \\
\hline Trab. RBC & $\begin{array}{l}\text { Trabajo inédito de Félix Varela, enviado a la } \\
\text { Revista Bimestre Cubana }\end{array}$ \\
\hline
\end{tabular}

\section{Referencias}

Baró, J. (1987). Glosario completo de los Milagros de Nuestra Señora de Gonzalo de Berceo. Boulder, Colorado : Society of Spanish and Spanish-American Studies, 1987.

Biblioteca Nacional (España). Ariadna [en línea]: catálogo automatizado de la Biblioteca

Nacional. [Madrid]: Biblioteca Nacional. URL: <telnet://ariadna.bne.es>, login: 'bn' [Consulta: 13 ene. 1999].

Catálogo temático de pensamientos de José Martí. Sala Cubana. Biblioteca Nacional José Martí.

Currás, E. (1995). Concierto y desconcierto en la organización del conocimiento actual y su intersección con el mundo de la información. // Scire. 1 : 1 (1995) 3-8.

Epigr : Epigrafiario de la Biblioteca Nacional [en disco] (1998). La Habana : Biblioteca Nacional José Martí, 1998.

Ibarra Martín, F. (1998). Metodología de la investigación social. La Habana : Editorial Pueblo y Educación, 1998. 
Maniez, J. (1992) Los lenguajes documentales y de clasificación : concepción, construcción y utilización en los sistemas documentales. Madrid : Fundación Germán Sanchez Ruipérez, 1992.

Martí, J. (1983) Obras completas. Ed. crítica. La Habana : Centro de Estudios Martianos, 1983.

Real Academia Española (1994). Diccionario de la lengua española. Madrid, 1994.

Rosental, M.; Iudin, P.(1973). Diccionario filosófico. La Habana : Editora Política, 1973.

Soto Balbón, M. A. (1993). Manual de CDS/ISIS para usuarios y administradores. La Habana : Universidad de La Habana, Dir. de Información Científico-Técnica y Bibliotecología, 1993.

UNESCO. División de Desarrollo de Software y Aplicaciones (1990). Manual de referencia Mini-micro CDS/ISIS. París : Unesco, 1990.

Vizcaya Alonso, D. (1997a). Información : procesamiento de contenido. Rosario, Argentina : Ediciones Nuevo Parhadigma, 1997.

Vizcaya Alonso, D. (1997b). Lenguajes documentarios. Rosario, Argentina : Ediciones Nuevo Parhadigma, 1997.

Vare : Base de datos bibliográfica [en disco] (1997). La Habana : Biblioteca Nacional José Martí, 1997. 